\title{
La cirugía precoz acelera el alivio de la ciática con resultados al año similares al tratamiento conservador
}

\author{
Early surgery accelerates sciatic pain relief with similar outcomes at one year as conservative treatment
}

Peul W y col. N Engl J Med 2007; 356: 2245-56.

\section{Objetivo}

Comparar la eficacia en la ciática discal de la cirugía precoz (CP) con el tratamiento conservador prolongado (TCP) con eventual cirugía subsecuente.

\section{Diseño}

Ensayo clínico controlado aleatorizado con un año con seguimiento.

\author{
Lugar \\ Estudio multicéntrico, Holanda.
}

\section{Pacientes}

283 pacientes con ciática de seis a 12 semanas de duración debida a hernia discal comprobada por resonancia magnética (RM). Se excluyeron pacientes con síndrome de cola de caballo, déficit muscular, episodios similares en el año previo, cirugía espinal previa, canal estrecho lumbar, espondilolistesis, embarazo, o enfermedad severa coexistente.

\section{Intervención}

El CP incluyó 141 y el TCP 142 sujetos. En la rama CP, 16 pacientes mejoraron espontáneamente y no fueron operados. Se intervinieron quirúrgicamente 125 pacientes con una mediana de tiempo para la cirugía de 1,9 semanas. En la rama TCP se ofreció cirugía antes de los seis meses ante aumento del dolor, ausencia de respuesta a la medicación o aparición de déficit neurológico; y también por persistencia de la ciática más allá de los seis meses desde la alegorización, operándose de este grupo 55 pacientes (39\%) con una mediana de tiempo para la cirugía de 14,6 semanas.

\section{Medición de resultados principales}

Cuestionario de Discapacidad de Roland, escala analógica visual $^{\star}(E V A)$ para dolor en el miembro inferior y percepción del paciente acerca de la mejoría al año. Análisis por intención de tratar*. $^{*}$.

\section{Resultados principales}

El puntaje de discapacidad no mostró diferencias significativas al año. Los asignados a CP refirieron alivio más rápido del dolor $(\mathrm{p}<0,001)$ y una más rápida recuperación (HR 1,97; IC95\% 1,72 a 2,22). La mediana de tiempo para la recuperación fue cuatro semanas (IC95\% 3,7-4,4) en el grupo CP; y 12,1 (IC95\% 9,5-14,9) en el TCP. Este efecto decayó con el tiempo y en ambos la probabilidad de recuperación percibida al año fue de $95 \%$. La pérdida de pacientes fue de $2 \%$ en ambos grupos.

\section{Conclusiones}

La CP acelera el alivio de la ciática y sus resultados al año son similares al TCP.

Palabras clave: Ciática, hernia de disco, cirugía

Key words: Sciatica, lumbar disc, surgery.

Fuente de financiamiento: Subsidio de la Organización de los Países Bajos para la Investigación y Desarrollo en Salud y la Fundación Hoelen, La Haya.

\section{Comentario}

La incidencia de ciática es aproximadamente cinco casos/1000 adultos/año, siendo la hernia discal la causa más frecuente. Esta problemática tiene impacto en la salud pública debido a los costos hospitalarios y ausentismo laboral consecuentes. La historia natural de la ciática discal es en general favorable, con resolución dentro de las ocho semanas en la mayoría de los pacientes ${ }^{1}$. Clásicamente la cirugía se ofrece en caso de dolor radicular persistente luego de haber realizado tratamiento conservador. Los resultados y el bajo riesgo de la microdiscectomía lumbar han sido reportados, así como también recientemente se han publicado trabajos comparando cirugía versus tratamiento conservador ${ }^{1-4}$.

La originalidad del trabajo de Peul y col. radica en que es el primer estudio que se propone evaluar de qué manera el momento de la cirugía influye en los resultados, contribuyendo en la determinación de su momento oportuno. Sus resultados proporcionan un punto de referencia para el proceso de toma de decisiones, aunque el hecho de que el ensayo no sea ciego puede disminuir la validez de las conclusiones.

\section{Conclusiones del comentador}

Los pacientes deben ser informados adecuadamente acerca de la historia natural y de los puntos relevantes para la toma de decisiones: manejo del dolor ciático y tiempo probable de recuperación con CP y con TCP. Quienes no toleren el dolor y/o que encuentren el curso natural de la recuperación inaceptablemente lento seguramente optarán por CP. Quienes controlen el dolor aceptablemente pueden decidir posponer la cirugía con la expectativa de que tal vez no la necesiten, sin que ello reduzca sus probabilidades de recuperación completa a un año. Ambas estrategias parecen ofrecer las mismas chances de recuperación a largo plazo, pero la CP continúa siendo una opción válida para el alivio rápido del dolor y acelerar la recuperación.

Ver glosario*

Alejandra Rabadán [ Servicio de Neurocirugía, Hospital Italiano de Buenos Aires. División Neurocirugía, Instituto de Investigaciones Médicas Alfredo Lanari Universidad de Buenos Aires. ]

Rabadán AT. Más rápido alivio de la ciática con cirugía precoz versus tratamiento conservador prolongado, y similar resultado al año. Evid. actual. práct. ambul; 10(4):107, Jul-Ago 2007. Comentado de: Peul WC y col. Surgery versus Prolonged Conservative Treatment for Sciatica. N Engl $J$ Med 2007 May 31; 356(22): 2245-2256. PMID: 17538084

\footnotetext{
Referencias

1. Awad JN y col.Clin Orthop Relat Res 443: 183-197, 2006

2. Carragaee EJ y col. J Bone Joint Surg Am 85: 102-108, 2003.

3. Osterman $\mathrm{H}$ y col. Spine 31: 2409-14, 2006.
}

4. Weinstein JN y col. JAMA 296: 2441-50, 2006 